\title{
Muskelfunktionsdiagnostik nach Janda
}

\author{
Sandra Krüger
}

REHA Bergmannstraße $5 \mathrm{GmbH}$, Berlin

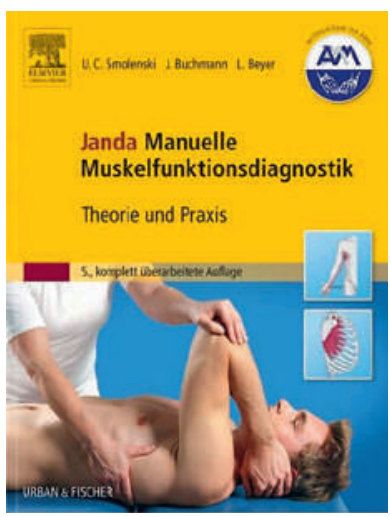

Ulrich-Christian Smolenski, Johannes Buchmann, Lothar Beyer u.a.

Janda Manuelle Muskelfunktionsdiagnostik Theorie und Praxis

München: Urban \& Fischer Verlag / Elsevier GmbH; 5. Auflage 2016.

312 Seiten. 53.60 CHF.

ISBN 978-3437464317

Namen- und Ideengeber des Buches ist der tschechische Neurologe und Psychiater Dr. Vladimir Janda, der intensive klinische Forschung zur Pathogenese und Therapie von chronischen muskuloskelettalen Schmerzen betrieb. Sein erstes Buch über Muskeltests und -funktionen schrieb er $1949 \mathrm{im}$ Alter von 21 Jahren. Jandas Ideen und Konzepte wurden inzwischen weiterentwickelt und sind fester Bestandteil der manuellen Medizin.

Das erste Kapitel befasst sich mit Physiologie und Pathophysiologie der Muskelfuktionen. Jedoch werden muskelphysiologische Grundkenntnisse in dem Buch teilweise schon vorausgesetzt. Die klinische Anwendung der Muskelphysiologie steht im Vordergrund. Das Augenmerk wird auf die tonischen Anteile der Sensomotorik gelenkt, um die Erkenntnisse aus den neurophysiologischen Zusammenhängen für manualdiagnostische und -therapeutische Überlegungen nutzen zu können.

Korrespondenz:
Die Skelettmuskulatur reagiert unter pathologischen Bedingungen mit bestimmten strukturellen Verände- rungen, so dass sich aus der Strukturpathologie des Skelettmuskels Hinweise auf zugrundeliegende Erkrankungen ableiten können. Der Strukturpathologie des Skelettmuskels widmet sich das zweite Kapitel. Im dritten Kapitel werden die Funktionspathologien des Skelettmuskels und deren Untersuchungsmethoden sowie manuelle Behandlungstechniken aufgeführt.

Das vierte und umfangreichste Kapitel trägt den Titel «Muskelfunktionstest - Testung der Kraft». Nach einer kurzen Einführung werden Muskelfunktionstests für alle Muskeln gegliedert nach anatomischen Gesichtspunkten (Gesicht, Hals und Körperstamm, obere Extremität und untere Extremität) erklärt und mit anatomischen Zeichnungen und Fotografien untermalt. Es werden jeweils die Ausgangsstellung, die Fixationspunkte und die zu erwartende Bewegung in den einzelnen Kraftstufen beschrieben. Das Einbeziehen von Triggerpunkten, Fernpunkten und Schmerzprojektionen verdeutlicht die Untersuchungsmethoden und die daraus abgeleiteten Behandlungsprizipien. In einer Tabelle finden sich jeweils als Übersicht die Hauptmuskeln, die die getestete Bewegung bewirken inklusive Ursprung, Ansatz und Innervation sowie gegebenenfalls Hilfsmuskeln, Neutralisationsmuskeln oder Stabilisationsmuskeln. Hinweise auf mögliche Fehler in der Testdurchführung haben grossen praktischen Nutzen.

Weitere Muskelfunktionstests bestehen in der Prüfung des Spannungsverhaltens, dem widmet sich das fünfte Kapitel.

Der visuellen Untersuchung von Stand und Gang ist wegen ihrer grossen orientierenden Bedeutung für die manualmedizinische klinische Untersuchung ein eigenes, sechstes Kapitel gewidmet.

Das siebte Kapitel geht auf die Hypermobilität und Besonderheiten bei der Untersuchung der konstitutionellen Beweglichkeit ein.

Für Ärzte, die sich mit dem muskuloskelettalen System beschäftigen, in Orthopädie, Neurologie und Rehabilitation sowie auch für Physiotherapeuten ist das Buch eine gute Hilfe in der Praxis. 